\title{
Etiologies of Hemodynamically Significant Aortic Regurgitation and Coronary Cusp Bending: Evaluation by Transesophageal Echocardiography
}

\author{
Naoki Hoshino $^{1}$, Akira Yamada ${ }^{1}$, Meiko Hoshino ${ }^{1}$, Yuka Kawada ${ }^{1}$, Sayuri Yamabe ${ }^{1}$, \\ Kayoko Takada ${ }^{1}$, Kunihiko Sugimoto ${ }^{2}$, and Hideo Izawa ${ }^{1}$ \\ ${ }^{1}$ Fujita Health University \\ ${ }^{2}$ Fujita Health University Hospital
}

April 15, 2021

\begin{abstract}
Background: While the number of patients with aortic regurgitation (AR) has been increasing in the aging society, its etiologies remain to be fully elucidated. Methods: We studied consecutive 126 patients with chronic moderate or severe AR who underwent TEE. After the study subjects were divided into 2 groups by the age of 65 years, AR etiology was examined in each group. Results: In the older group $(\mathrm{n}=85)$, cusp bending was the most frequent cause of AR $(48.2 \%)$, and right coronary cusp (RCC) was the most common (90.2\%). In the younger group $(\mathrm{n}=41)$, bicuspid valve was the most frequent cause (36.5\%), and cusp bending was less frequent (16.5\%). Multivariate analysis revealed that age was the only factor associated with cusp bending. Conclusion: Cusp bending of RCC was the most frequent etiology of AR in the elderly. Because AR caused by cusp bending has a possibility to be mended by aortic plasty, it is important to detect it by TEE especially in the older patients.
\end{abstract}

\section{Etiologies of Hemodynamically Significant Aortic Regurgitation and Coronary Cusp Bending: Evaluation by Transesophageal Echocardiography}

Naoki Hoshino ${ }^{1}$, Akira Yamada ${ }^{1}$, Yuka Kawada ${ }^{1}$, Meiko Hoshino ${ }^{1}$, Sayuri Yamabe ${ }^{1}$, Kayoko Takada ${ }^{3}$, Kunihiko Sugimoto ${ }^{2}$, Hideo Izawa ${ }^{1}$

1. Department of Cardiology, Fujita Health University School of Medicine, Toyoake, Japan

2. Clinical Laboratory, Fujita Health University Hospital, Toyoake, Japan

3. School of Health Sciences, Fujita Health University, Toyoake, Japan

Corresponding author: Akira Yamada

Address: 1-98 Dengakugakubo, Kutsukake-cho, Toyoake, Aichi 470-1192, JAPAN

business telephone numbers: +81562932312

email address:a-yamada@fujita-hu.ac.jp

\section{ABSTRACT}

Background: While the number of patients with aortic regurgitation (AR) has been increasing in the aging society, its etiologies remain to be fully elucidated. We examined the causes of AR in the elder patients by analyzing transesophageal echocardiography (TEE) images. 
Methods: We studied consecutive 132 patients with chronic moderate or severe AR who underwent TEE for further evaluation of AR. After the study subjects were divided into 2 groups by the age of 65 years, AR etiology, which was confirmed based on the TEE findings, was investigated in each group.

Results: The total number of patients eligible for this study was 126 (mean age $67 \pm 12$ years), consisting of the older group $(\mathrm{n}=85$, mean age $74 \pm 5$ years $)$ and the younger group $(\mathrm{n}=41$, mean age $52 \pm 11$ years). In the older group, cusp bending was the most frequent cause of AR (48.2\%), and right coronary cusp (RCC) was the most common region to be affected (90.2\%). On the other hand, in the younger group, bicuspid aortic valve was the most common etiology (36.5\%), and cusp bending accounted for only $19.5 \%$ in this group. Subsequently, all the study subjects were re-classified into 2 groups with or without cusp bending. Between these 2 groups, only age showed a significant difference $(\mathrm{p}=0.0017)$, and multivariate analysis revealed that age was the only factor associated with cusp bending.

Conclusion: Cusp bending of RCC was the most frequent etiology of AR in the elderly. Because AR caused by cusp bending has a possibility to be mended by aortic plasty, it is important to detect it especially in the older patients.

Keywords: valvular heart disease, aortic regurgitation, cusp bending

\section{INTRODUCTION}

The prevalence of valvular heart diseases (VHD) is estimated to be $2.5 \%$ in developed countries (1). Among $\mathrm{VHD}$, aortic regurgitation (AR) is reported to be the third most common form, following mitral regurgitation (MR) and aortic stenosis (AS) (2). The prevalence of VHD increases especially in patients over 65 years of age because of the predominance of degenerative etiologies (1). Several surveys have been performed to clarify the etiologies of AR so far. Roberts et al. examined 268 patients who had isolated aortic valve (AV) replacement (AVR) for pure AR and concluded that the causes of AR were diverse and the most common category was "cause unclear" in their study (3). In the Euro Heart Survey on VHD, degenerative tricuspid and bicuspid AR were the most common etiologies, accounting for approximately two-thirds of the underlying etiology of AR in the Euro Heart Survey on VHD (4) (5).

In detail, degenerative origin of AR accounted for approximately half (50.3\%) and rheumatic AR was present in $15.2 \%$. In addition, Braun et al. reported that RA and other connective tissue diseases can cause valvular disease including AR (6). While AVR has been an established surgical definitive therapy for the treatment of AR, aortic valvuloplasty (AVP) has also been acknowledged gradually as another surgical option for AR (7). Although AVP is not so prevailed as mitral valvuloplasty, both plasty procedures have more advantages such as freedom from anticoagulant therapy. It is essential to conduct precise anatomical assessment preoperatively for a successful valvuloplasty and for the prevention of recurrence of significant regurgitation (8). Recent advancement in echocardiography enables to detect more detailed abnormal morphology of aortic cusps accurately. Cusp bending of aortic valve has been detected more commonly with AR on transesophageal echocardiography (TEE) examination, which is observed as a fibrous band on a cusp (Figure 1, 2, 3, 4) (9) (10) (11). Nevertheless, the prevalence of cusp bending and its clinical characteristics remain to be clarified in pure AR patients. Therefore, the aim of this study was to thoroughly examine the etiologies of AR through observing aortic valve morphology by use of TEE images.

\section{Study Population}

We retrospectively studied consecutive 146 patients with chronic moderate or severe AR who underwent TEE for further evaluation of AR in our institution between April 2013 and April 2020. The exclusion criteria were as follows: age of less than 18 years, acute AR, previous history of ascending aortic replacement and/or aortic valve plasty, concomitant moderate or severe AS, evident previous history of infective endocarditis (IE) on any aortic cusp, and poor echo images. We collected the patients' clinical information by reviewing their medical charts.

Transthoracic and Transesophageal Echocardiography 
Transthoracic echocardiography (TTE) examinations were performed with a commercially available machine (IE33 or EpiQ CVx, Philips Medical System, USA) ultrasound system equipped with a 2- to 3.5-MHz transducer in the left lateral decubitus position. On top of conventional echo measurements, AR severity was evaluated comprehensively and graded in 3 grades (mild, moderate, severe) in accordance with the guidelines of the American Society of Echocardiography (12) (13). The study subjects of this study were those with moderate or severe AR. In addition, after a consent form of TEE was obtained from every patient, TEE was performed with a transducer (X7-2t or X8-2t) for further evaluation of AR within 3 months after the TTE examination. 2D and 3D TEE grayscale and color images were acquired at the level of the aortic valve and root in all the patients. All images were stored digitally and transferred onto a dedicated image server for subsequent review.

\section{Assessment of AR Etiologies}

The recorded echocardiographic images were reviewed off-line for the assessment of AR etiologies by 2 experienced observers who had no medical information about the patients beforehand. Aortic cusp bending was defined as a fibrous band on a cusp on TEE images (Figure 1-1, 2 ).

\section{Statistical Analysis}

As to continuous data, they are expressed as mean values \pm standard deviation (SD) if they meet normal distribution. If not, their median and inter-quartile ranges are described. All data were compared by Student's t-test or Wilcoxon rank sum test whenever appropriate. On the other hand, categorical data were presented as percentages and compared by chi-square or Fisher exact test. Univariate and multivariate regression analyses were used to identify clinical variables that were associated with aortic cusp bending. JMP ver12.0 (SAS Institute, Cary, USA) was used for statistical analyses. A p value of $<0.05$ was considered statistically significant.

\section{RESULTS}

\section{Patients Characteristics}

Based on the exclusion criteria, 20 patients were excluded from this study (2 cases of ascending aortic replacement and/or aortic valvuloplasty, 2 cases of moderate or severe AS, 16 cases of IE). In total, 126 patients were eligible for this study (mean age $67 \pm 12$ years, male $73 \%$ ). Table 1 shows their baseline clinical characteristics, comorbidities and echocardiographic measurements. Three fourths of the study subjects were hypertensive, and $43 \%$ of them had chronic kidney disease (CKD) (estimated glomerular filtration rate $<60$ $\mathrm{ml} / \mathrm{min} / 1.73 \mathrm{~m}^{2}$ ). Thirteen $(10 \%)$ patients had a connective tissue disease (rheumatic arthritis 5 , systemic lupus erythematosus 2, Marfan syndrome 1, Takayasu arteritis 1, autosomal dominant polycystic kidney disease 2, polymyalgia rheumatica 1, Behçet's disease 1). More than half of them had severe AR, resulting in their dilated left ventricular (LV) size (LV end-diastolic diameter: $60 \mathrm{~mm}$ ) with slightly reduced LV ejection fraction $(51 \%)$ as well as slightly dilated sinus of Valsalva $(38 \mathrm{~mm})$ (median values, respectively).

Etiologies of AR (Table 2)

In all the study subjects, the most common etiology of AR was cusp bending (33.0\%), followed by aortic root enlargement $(22.2 \%)$, and bicuspid AV (17.4\%). Seven (5.5\%) patients had both AV cusp bending and aortic root enlargement. Even normal finding was also noted in $7.9 \%$ of the subjects. In AV cusp bending, right coronary cusp (RCC) was the most affected (90.5\%, 38 out of 42 cusp bending cases).

\section{Comparison between Cusp Bending and Non-Cusp Bending Groups(Table 3)}

As the next step, all the study subjects were classified into 2 groups with or without aortic cusp bending on any cusp. The number of patients with cusp bending was 49 (mean age $72 \pm 7$ years), whereas that of those without cusp bending was 77 (mean age $64 \pm 14$ years). The age was significantly higher in the cusp bending group $(\mathrm{p}=0.0017)$. While the patients with aortic cusp bending had significantly lower height (159 vs 165 $\mathrm{cm}$, median, $\mathrm{p}=0.0037)$ and less body surface area (1.58 versus $1.63 \mathrm{~m}^{2}$, median, $\left.\mathrm{p}=0.0287\right)$, LV echo measurements did not differ between the 2 groups. However, those with cusp bending had smaller diameters 
of Valsalva sinus (36 vs $39 \mathrm{~mm}$, median, $\mathrm{p}=0.0188$ ), ST junction (30 vs $33 \mathrm{~mm}$, median, $\mathrm{p}=0.0150$ ) and mid-ascending aorta ( $35 \mathrm{vs} 38 \mathrm{~mm}$, median, $\mathrm{p}=0.0097$ ). In addition, patients with mitral prolapse were observed only in the cusp-bending group ( $8 \%$ vs $0 \%, \mathrm{p}=0.0053)$.

\section{AR Etiologies According to Age (Table 4)}

Subsequently, all the subjects were re-classified into 2 groups based on their age at 65 years: the elderly group $(\mathrm{n}=85$, mean age $74 \pm 5$ years $)$ and the younger group $(\mathrm{n}=41$, mean age $52 \pm 11$ years $)$. In the elderly group, cusp bending was the most frequent cause of AR (48.2\%), and RCC was the most affected cusp (90.2\%). On the other hand, in the younger group, bicuspid AV was the most common etiology (36.5\%), and cusp bending accounted for only $19.5 \%$ in this group.

To examine the contributing factors to aortic cusp bending, logistic regression analysis was performed with age, hypertension, diabetes, CKD and connective tissue diseases included as dependent variables, which revealed that age was the only factor associated with cusp bending (Table 5).

\section{DISCUSSION}

We showed that:

In AR patients over 65 years of age, aortic cusp bending was the most frequent AR etiology. On the other hand, in the younger patients, bicuspid AV was the most observed cause of AR.

Aging was the only significant factor to predict coexisting cusp bending of AV.

Cusp bending could occur in any AV cusp, nevertheless, it was detected on RCC by far the most frequently (45 out of 49 cusp-bending related cases). This was consistent with the previous study, reporting that $70 \%$ of the isolated cusp bending was seen on RCC (9). Roberts et al. reported that the most frequent cause of AR was "unclear", accounting for 34\% (3). The characteristics of the AR patients with "etiology unclear" were higher age with atherosclerotic diseases, in common with the cusp-bending group in this study. Higher age may also contribute to the result that the patients in this group were shorter in height with smaller body surface area. Recent improvement in TEE image quality has helped to detect cusp-bending more clearly, which may have been included in "etiology unclear". Aging is associated with degenerative changes in cusps in various signaling pathways of molecular levels (14). In this study, however, there were no differences between those with and without cusp-bending in terms of the prevalence of atherosclerotic factors such as hypertension, diabetes, lipid disorder, CKD and smoking. Most of the patients in this study had various atherosclerotic diseases originally, and aging is considered the most influential contributor to occurrence of cusp bending above all. Although the number of patients with mitral prolapse was small (4 of 126 patients), they were detected only in cusp bending group, which may be associated with valvular vulnerability in patients with cusp bending.

There are several classifications of AR, and adaptation of the Carpentier classification originally designed for the mitral valve have been described for AR and can be helpful to understand the mechanism of AR, to guide valve repair technique, and to predict recurrence of AR (15). This scheme classifies dysfunction based on the aortic root and leaflet morphology. Cusp bending belongs to type II, cusp prolapse, in this category. As shown in this report, AV cusp bending may have favorable surgical repairability and outcome such as freedom from bleeding events caused by anti-coagulants or IE (9). Therefore, it is important to detect cusp bending preoperatively.

There are some limitations in the present study. This is a single center study and the number of patients enrolled in this study was relatively small. Secondly, 10 cases (7.9\%) were classified as echocardiographically normal finding (Table 2). Even without any abnormality on AV cusps, AR can be generated from imbalanced sizes of aortic annulus, Valsalva sinus and ST junction (16) (17), which may be beyond echocardiographic resolution capability. Further investigation will be required for the validation of our study.

\section{CONCLUSIONS}


Cusp bending of RCC was the most frequent etiology of AR in the elderly. Because AR caused by cusp bending has a possibility to be repaired by AVP, it is important to identify the mechanism responsible for AR especially in the older patients.

\section{REFERENCES}

1) Iung B, Vahanian A. Epidemiology of acquired valvular heart disease. Can J Cardiol. 2014 Sep;30(9):962970.

2) Steven M Hollenberg. Valvular Heart Disease in Adults: Etiologies, Classification, and Diagnosis. FP Essent. 2017 Jun;457:11-16.

3) Roberts WC, Ko JM, Moore TR, et al Causes of pure aortic regurgitation in patients having isolated aortic valve replacement at a single US tertiary hospital (1993 to 2005). Circulation. 2006 Aug 1;114(5):422-429.

4) Iung B, Baron G, Butchart EG, et al A prospective survey of patients with valvular heart disease in Europe: The Euro Heart Survey on Valvular Heart Disease. Eur Heart J. 2003 Jul;24(13):1231-1243.

5) Baumgartner H, Falk V, Bax JJ, et al 2017 ESC/EACTS Guidelines for the management of valvular heart disease. Eur Heart J. 2017 Sep 21;38(36):2739-2791.

6) Braun J, Krüger K, Manger B et al Cardiovascular Comorbidity in Inflammatory Rheumatological Conditions. Dtsch Arztebl Int. 2017 Mar 24;114(12):197-203.

7) Rimmer L, Ahmad MU, Chaplin G, et al Aortic Valve Repair: Where Are We Now? Heart Lung Circ. $2019 \mathrm{Jul} ; 28(7): 988-999$.

8) Kunihara T. Aortic valve repair for aortic regurgitation and preoperative echocardiographic assessment. J Med Ultrason (2001). 2019 Jan;46(1):51-62.

9) Boodhwani M, de Kerchove L, Watremez C, et al Assessment and repair of aortic valve cusp prolapse: implications for valve-sparing procedures. J Thorac Cardiovasc Surg. 2011 Apr;141(4):917-925.

10) Shibayama K, Watanabe H, Murai T, et al Aortic regurgitation caused by cusp bending of aortic valve leaflet. J Echocardiogr. 2012 Mar;10(1):21-23.

11) Soga F, Takaya T, Mori S, et al Bending of the aortic valvar leaflet causing severe aortic regurgitation in a patient with osteogenesis imperfecta. Eur Heart J Cardiovasc Imaging. 2016 Jun;17(6):708.

12) Lang RM, Badano LP, Mor-Avi V, Afilalo J, Armstrong A, Ernande

L, Flachskampf FA, Foster E, Goldstein SA, Kuznetsova T, Lancellotti P, Muraru D, Picard MH, Rietzschel ER, Rudski L, Spencer KT, Tsang W, Voigt JU. Recommendations for cardiac chamber quantification by echocardiography in adults: an update from the American Society of Echocardiography and the European Association of Cardiovascular Imaging. J Am Soc Echocardiogr 2015;28:1-39.e14.

13) Zoghbi WA, Adams D, Bonow RO, et al Recommendations for Noninvasive Evaluation of Native Valvular Regurgitation: A Report from the American Society of Echocardiography Developed in Collaboration with the Society for Cardiovascular Magnetic Resonance. J Am Soc Echocardiogr. 2017 Apr;30(4):303-371.

14) Costantino S, Paneni F, Cosentino F. Ageing, metabolism and cardiovascular disease. J Physiol. 2016 Apr 15;594(8):2061-2073. 
15) Boodhwani M, de Kerchove L, Glineur D, et al Repair-oriented classification of aortic insufficiency: impact on surgical techniques and clinical outcomes. J Thorac Cardiovasc Surg. 2009 Feb;137(2):286-294.

16) Marom G, Haj-Ali R, Rosenfeld M, et al Aortic root numeric model: correlation between intraoperative effective height and diastolic coaptation. J Thorac Cardiovasc Surg. 2013 Jan;145(1):303-304.

17) Marom G, Haj-Ali R, Rosenfeld M, et al Numerical model of the aortic root and valve: optimization of graft size and sinotubular junction to annulus ratio. J Thorac Cardiovasc Surg. 2013 Nov;146(5):1227-1231.

Table 1 Baseline Characteristics and Echocardiographic Parameters in All Patients

\begin{tabular}{ll}
\hline Clinical Characteristics & \\
\hline Age, yrs & $67 \pm 12$ \\
Male, n (\%) & $93(73.8)$ \\
Height, cm & $163[155-168]$ \\
Body Weight, kg & $58[49-65]$ \\
Body Mass Index, kg/m² & $21[19-24]$ \\
Body Surface Area, m² & $1.61[1.47-1.75]$ \\
Smoking, n (\%) & $67(53.1)$ \\
Comorbidity & \\
Hypertension, n (\%) & $95(75.3)$ \\
Dyslipidemia, n (\%) & $47(37.3)$ \\
Diabetes, n (\%) & $17(13.4)$ \\
Previous Ischemic Heart Disease, n (\%) & $25(19.8)$ \\
Atrial Fibrillation, n (\%) & $28(22.2)$ \\
Connective Tissue Disease, n (\%) & $13(10.3)$ \\
Mitral Prolapse, n (\%) & $4(3.1)$ \\
Steroid User, n (\%) & $7(5.5)$ \\
Maintenance Dialysis, n (\%) & $2(1.5)$ \\
Chronic Kidney Disease, n (\%) & $55(43.6)$ \\
eGFR, ml/min/1.73m ${ }^{2}$ & $57[42-70]$ \\
Echocardiographic Parameters & \\
LVEDD, mm & $60[53-66]$ \\
LVESD, mm & $46[38-53]$ \\
LVEF, \% & $51[38-59]$ \\
IVSD, mm & $10[9-11]$ \\
PWD, mm & $10[9-11]$ \\
Aortic Root Dimensions & \\
Annulus Diameter, mm & \\
Sinus of Valsalva Diameter, mm & $38[21-26]$ \\
ST Junction Diameter, mm & $31[28-43]$ \\
Mid-Ascending Aorta Diameter, mm & $37[33-42]$ \\
AR Severity & \\
Moderate, n (\%) & $29(23.0)$ \\
Moderate to Severe, n (\%) & $27(21.4)$ \\
Severe, n (\%) & $70(55.6)$ \\
\hline & \\
& \\
&
\end{tabular}

LVEDD: Left Ventricular End-Diastolic Diameter, LVESD: Left Ventricular End-Systolic Diameter, LVEF: Left Ventricular Ejection Fraction, IVSD: Interventricular Septal Diameter, PWD: Posterior Wall Diameter.

Table 2 AR Etiologies in All Patients 


\begin{tabular}{ll}
\hline \multicolumn{1}{c}{ Coronary Cusp Bending, n (\%) } & $42(33.0)$ \\
\hline RCC, n (\%) & $36(28.5)$ \\
LCC, n (\%) & $2(1.5)$ \\
NCC, n (\%) & $2(1.5)$ \\
RCC+NCC, n (\%) & $2(1.5)$ \\
Aortic Root Enlargement, n (\%) & $28(22.2)$ \\
Aortic Root Enlargement + RCC Bending, n (\%) & $7(5.5)$ \\
Bicuspid Aortic Valve, n (\%) & $22(17.4)$ \\
Coaptation Failure Due to Calcification of Cusps, & $10(7.9)$ \\
n (\%) & $10(7.9)$ \\
Normal Finding, n (\%) & $2(1.5)$ \\
Chronic Ascending Aortic Dissection, n (\%) & $1(0.7)$ \\
Quadricuspid Aortic valve, n (\%) & $1(0.7)$ \\
RCC Flail, n (\%) & $1(0.7)$ \\
RCC+LCC Prolapse, n (\%) & $1(0.7)$ \\
RCC Prolapse Due to VSD, n (\%) & $1(0.7)$ \\
Restrictive RCC with Membranous Interventricular & \\
Septal Aneurysm, n (\%) & $126(100)$ \\
Total, n (\%) &
\end{tabular}

RCC: Right Coronary Cusp, LCC: Left Coronary Cusp, NCC: Non Coronary Cusp, VSD: Ventricular Septal Defect.

Table 3 Comparison of Baseline Characteristics and Echocardiographic Parameters between "Cusp bending" and "Non Cusp Bending" Patients

Cusp Bending $(\mathrm{n}=49,38.5 \%) \quad$ Non Cusp Bending $(\mathrm{n}=77,61.5 \%) \quad \mathrm{P}$ value

Clinical Characteristics

Age, yrs

Male, n (\%)

Height, cm

Body Weight, kg

Body Mass Index, $\mathrm{kg} / \mathrm{m}^{2}$

Body Surface Area, $\mathrm{m}^{2}$

Smoking, n (\%)

Comorbidity

Hypertension, n (\%)

Dyslipidemia, n (\%)

Diabetes, n (\%)

Previous Ischemic Heart Disease, n (\%)

Atrial Fibrillation, n (\%)

Steroid User, n (\%)

Connective Tissue Disease, n (\%)

Maintenance Dialysis, n (\%)

Mitral Prolapse, n (\%)

Chronic Kidney Disease, n (\%)

eGFR, $\mathrm{ml} / \mathrm{min} / 1.73 \mathrm{~m}^{2}$

Echocardiographic Parameters

LVEDD, mm
$72 \pm 7.1$

$35(71.4)$

159 [154-164]

54 [49-62]

21 [19-24]

1.58 [1.44-1.64]

$27(55.1)$

40 (81.6)

21 (42.8)

8 (16.3)

9 (18.3)

$14(28.5)$

$3(6.1)$

5 (10.2)

$1(2.0)$

4 (8.1)

22 (44.8)

55 [41-63]

62 [57-67]
$64 \pm 14$

58 (75.3)

165 [156-169]

59 [50-67]

21 [19-24]

1.63 [1.48-1.77]

$40(51.9)$

$55(71.4)$

$26(33.7)$

9 (11.6)

$16(20.7)$

14 (18.1)

4 (5.1)

$8(10.3)$

1 (1.2)

0 (0)

33 (42.8)

58 [42-75]

59 [51-64]
0.0017

0.6288

0.0037

0.0959

0.9880

0.0287

0.7293

0.1887

0.3049

0.4614

0.7820

0.1752

0.8255

0.9734

0.7442

0.0053

0.8219

0.2320

0.0919 


\begin{tabular}{llll}
\hline & Cusp Bending $(\mathrm{n}=49,38.5 \%)$ & Non Cusp Bending $(\mathrm{n}=77,61.5 \%)$ & P value \\
\hline LVESD, mm & $48[41-55]$ & $44[36-52]$ & 0.1250 \\
LVEF, \% & $49[35-56]$ & $53[39-60]$ & 0.0702 \\
IVSD, mm & $10[9-12]$ & $10[9-11]$ & 0.4623 \\
PWD, mm & $11[10-12]$ & $10[9-11]$ & 0.3924 \\
Aortic Root Dimensions & & & \\
Annulus Diameter, mm & $23[20-25]$ & $23[21-26]$ & 0.1275 \\
Sinus of Valsalva Diameter, mm & $36[33-40]$ & $39[33-47]$ & 0.0188 \\
ST Junction Diameter, mm & $30[27-34]$ & $38[34-44]$ & 0.0150 \\
Mid-Ascending Aorta Diameter, mm & $35[32-39]$ & & 0.0097 \\
AR Severity & $9(18.4)$ & $20(26.0)$ & \\
Moderate, n (\%) & $11(22.4)$ & $16(20.8)$ & \\
Moderate to Severe, n (\%) & $29(59.2)$ & & \\
Severe, n $(\%)$ & & & \\
\hline
\end{tabular}

LVEDD: Left Ventricular End-Diastolic Diameter, LVESD: Left Ventricular End-Systolic Diameter, LVEF: Left Ventricular Ejection Fraction, IVSD: Interventricular Septal Diameter, PWD: Posterior Wall Diameter.

Table 4

AR Etiologies in Older Patients ([?]65 years old)

\begin{tabular}{ll}
\hline Coronary Cusp Bending, n (\%) & $37(43.3)$ \\
\hline RCC, n (\%) & $31(36.4)$ \\
LCC, n (\%) & $2(2.3)$ \\
NCC, n (\%) & $2(2.3)$ \\
RCC+NCC, n (\%) & $2(2.3)$ \\
Aortic Root Enlargement, n (\%) & $17(20.0)$ \\
Coaptation failure due to calcification of the & $9(10.5)$ \\
leaflets & $8(9.4)$ \\
Normal Finding, n (\%) & $7(8.2)$ \\
Bicuspid Aortic Valve, n (\%) & $4(4.7)$ \\
Aortic Root Enlargement+RCC bending & $1(1.1)$ \\
Chronic Ascending Aortic Dissection, n (\%) & $1(1.1)$ \\
RCC Flail, n (\%) & $1(1.1)$ \\
Restrictive RCC with Membranous Interventricular & \\
Septal Aneurysm, n (\%) & $85(100)$ \\
Total, n (\%) &
\end{tabular}

RCC: Right Coronary Cusp, LCC: Left Coronary Cusp, NCC: Non Coronary Cusp.

AR Etiologies in Younger Patients ( $<65$ years old)

\begin{tabular}{ll}
\hline Bicuspid Aortic Valve, n (\%) & $15(36.5)$ \\
\hline Aortic Root Enlargement, n (\%) & $11(26.8)$ \\
Coronary Cusp Bending (RCC Bending), n (\%) & $5(12.1)$ \\
Aortic Root Enlargement + RCC Bending, n (\%) & $3(7.3)$ \\
Normal Finding & $2(4.8)$
\end{tabular}




\begin{tabular}{ll}
\hline Bicuspid Aortic Valve, n (\%) & $15(36.5)$ \\
\hline RCC+LCC prolapse & $1(2.4)$ \\
Coaptation failure due to calcification of the leaflets & $1(2.4)$ \\
RCC Prolapse Due to VSD, n (\%) & $1(2.4)$ \\
Quadricuspid Aortic Valve, n (\%) & $1(2.4)$ \\
Chronic Ascending Aortic Dissection, n (\%) & $1(2.4)$ \\
Total, n (\%) & $41(100)$ \\
\hline
\end{tabular}

RCC: Right Coronary Cusp, LCC: Left Coronary Cusp, VSD: Ventricular Septal Defect.

Table 5 Logistic Regression Analysis to Detect Independent Factors Associated with Cusp Bending

\begin{tabular}{llll}
\hline Factor & Odds Ratio & 95\%CI & $\mathrm{P}$ value \\
\hline Age, yrs & 1.07 & $1.03-1.13$ & 0.0014 \\
Hypertension, n (\%) & 1.28 & $0.49-3.43$ & 0.6096 \\
Diabetes, n (\%) & 1.73 & $0.55-5.45$ & 0.3420 \\
Chronic Kidney Disease, n (\%) & 0.62 & $0.27-1.40$ & 0.2546 \\
Connective Tissue Disease, n (\%) & 0.93 & $0.24-3.54$ & 0.9131 \\
\hline
\end{tabular}

CI: Confidence Interval.

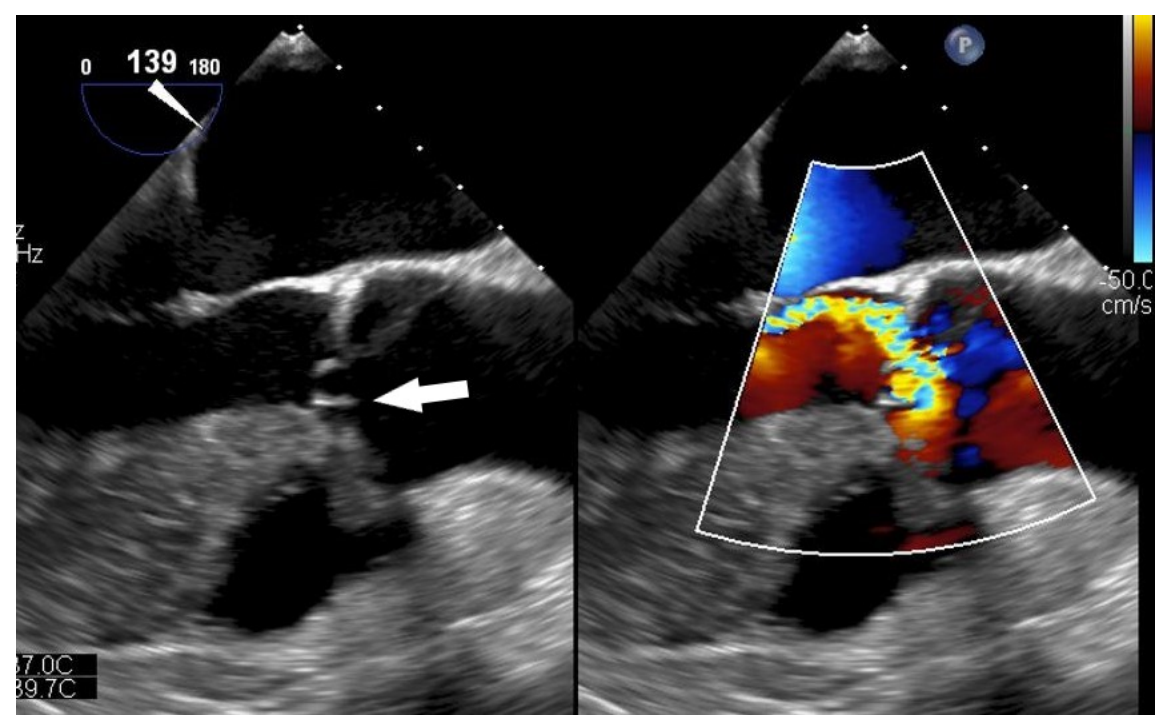



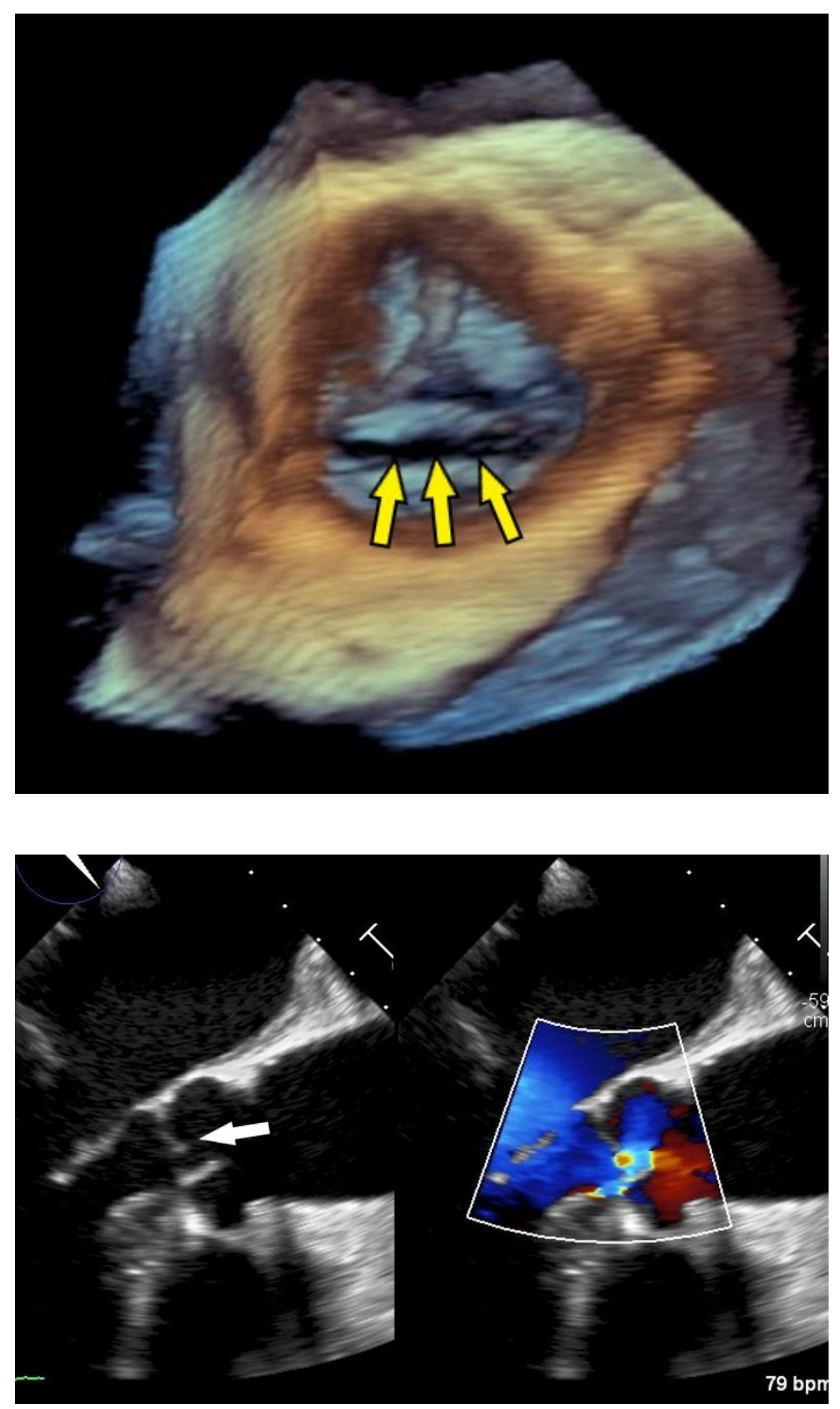

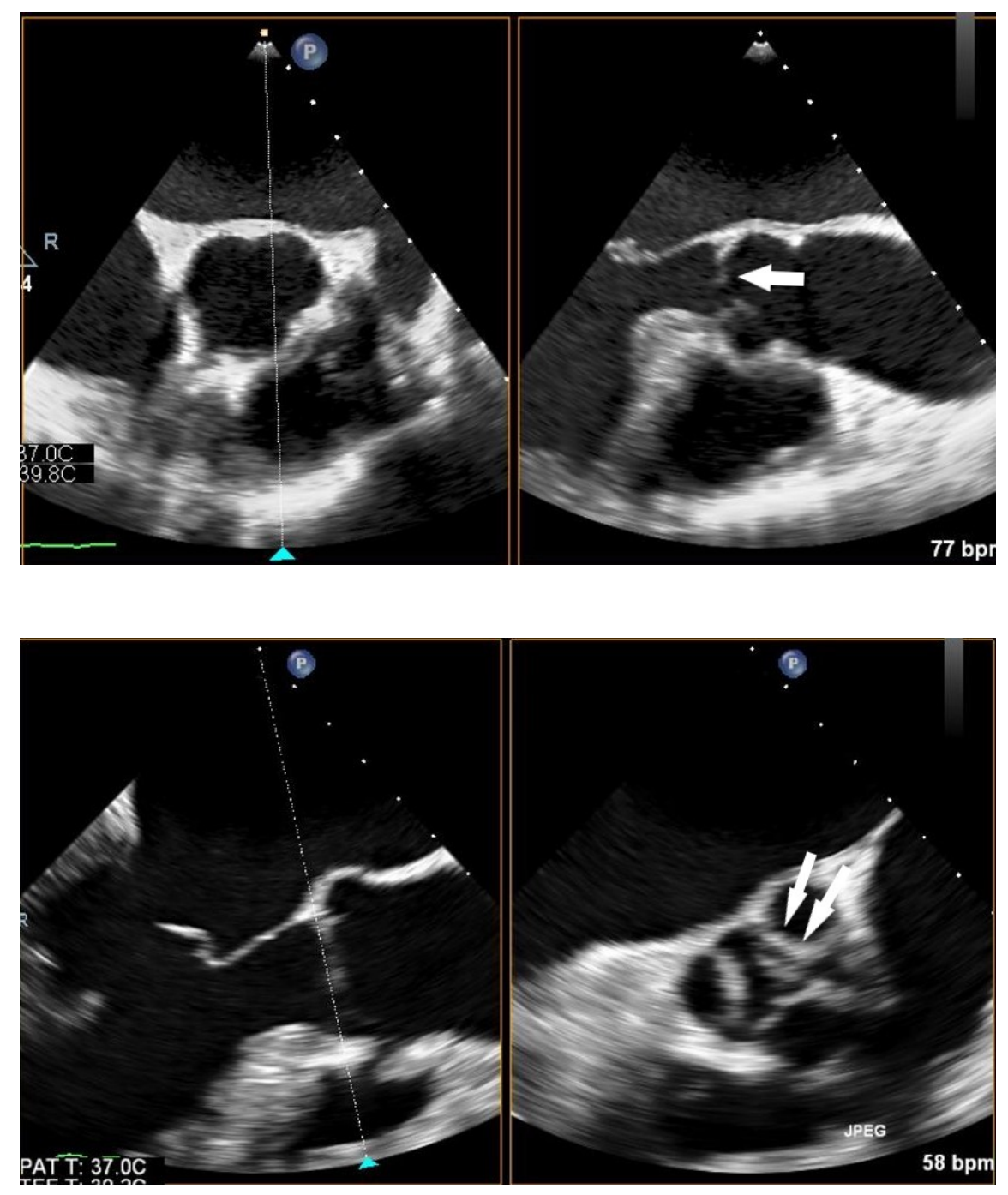

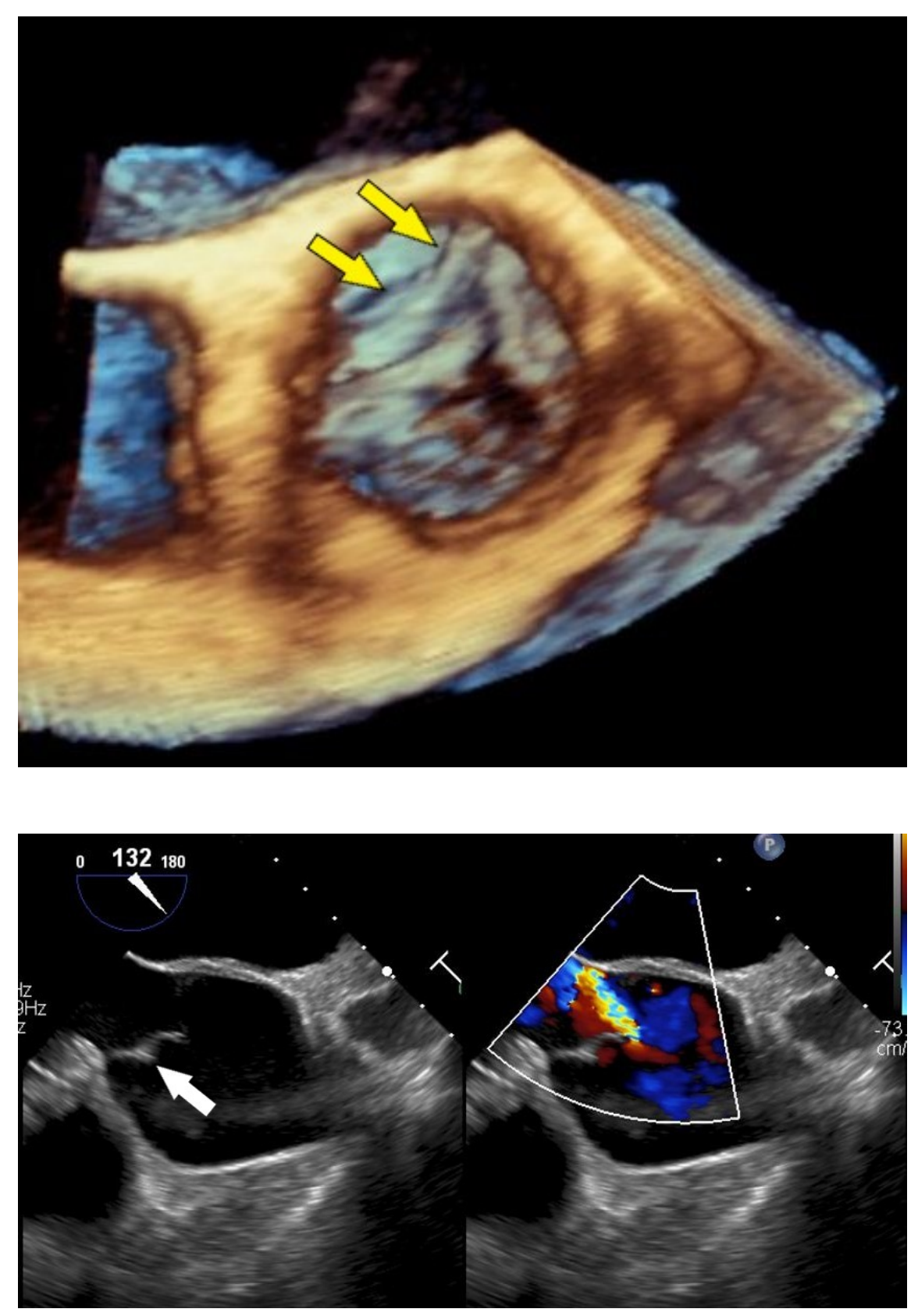


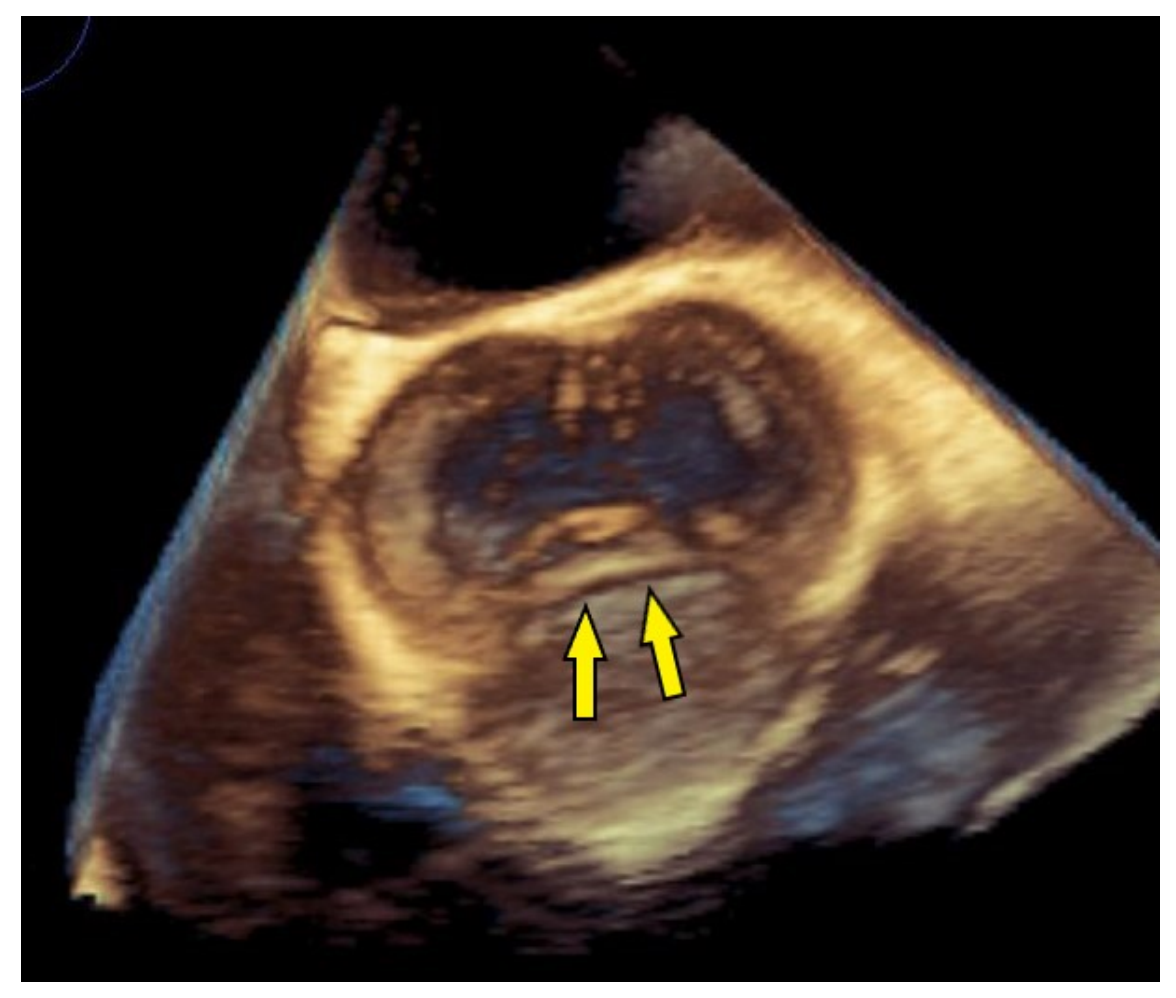

